\title{
A spindle cell squamous cell carcinoma on the cheek presenting with in-transit metastases and a satellite lesion
}

Eui-Tae Lee

Department of Plastic and

Reconstructive Surgery, Chungbuk

National University College of Medicine,

Cheongju, Korea
Spindle cell squamous cell carcinoma (SpSCC) is a biphasic tumor composed of squamous cell epithelial and spindle cell mesenchymal components, both of which are malignant. Cutaneous SpSCC can cause diagnostic and therapeutic difficulties because of its rarity, heterogeneity, morphological similarity to other cutaneous spindle cell neoplasms, and uncertain pathogenesis and prognosis, particularly when the squamous cell carcinoma component is minimal or missing. Intransit metastasis and satellite lesion (satellitosis) constitute a spectrum of non-nodal regional metastases. Here the author reports the first known case of cutaneous SpSCC presenting with intransit metastases and a satellite lesion, which were exceptionally aggressive. A 77-year-old female patient presented with a $3 \times 3 \times 0.5 \mathrm{~cm}$ mass on her right cheek. Despite wide excision and postoperative radiation, the patient resulted in local recurrence and multiple distant metastases within 3 months. If many high-risk factors-particularly satellitosis and in-transit metastases are observed in a tumor with epithelial to mesenchymal transition, then further wide excision and adjuvant chemoradiation should be considered early in the treatment process. A multidisciplinary approach could be the key to cure the most aggressive malignancies of the skin, as in other organs.

Keywords: Epithelial-mesenchymal transition / Neoplasms / Squamous cell

\section{INTRODUCTION}

Spindle cell squamous cell carcinoma (SpSCC), also known as sarcomatoid squamous cell carcinoma (SCC), is a biphasic variant of SCC consisted of a mesenchymal component of sarcomatoid spindle cell proliferation and an epithelial component of SCC [1]. Cutaneous SpSCC is very rare and only 11 cases have been reported in Korea [2-4]. It can cause diagnostic and therapeutic problems particularly when the SCC component is minimal or absent because of ulceration or necrosis.

\section{Correspondence: Eui-Tae Lee}

Department of Plastic and Reconstructive Surgery, Chungbuk National University College of Medicine, 1 Chungdae-ro, Seowon-gu, Cheongju 28644, Korea

E-mail: etlee@chungbuk.ac.kr

Received November 4, 2019 / Revised November 30, 2019 / Accepted December 10, 2019
In-transit metastasis and satellite lesion (satellitosis) mean intralymphatic or, less frequently, angiotrophic tumor spread; these terms differ only in the distance of spread. When a regional metastasis is within $2 \mathrm{~cm}$ (an arbitrary cutoff) from the tumor, it is defined as satellitosis, whereas a regional metastasis beyond $2 \mathrm{~cm}$ is defined as in-transit metastasis [5-7]. The concept and clinical significance of in-transit metastasis and satellitosis have been established for melanoma, but they are very rare in other skin malignancies; overall only 59 in-transit metastases from SCCs have been reported [8-11].

Here the author reports an exceptionally aggressive cutaneous case of SpSCC, in which the patient showed a satellite lesion and recurrent in-transit metastases. 


\section{CASE REPORT}

A 77-year-old woman presented with a $3 \times 3 \times 0.5 \mathrm{~cm}$ rubbery, hard, protruding mass on the right cheek. A small polyp developed 1 year ago and grew suddenly 1 month ago. It enlarged to $4 \times 5 \times 2 \mathrm{~cm}$ during 24 days of assessment and preparation for surgery. Computed tomography (CT) displayed a $2.5 \mathrm{~cm}$ irregular highly enhanced lesion. The preoperative work-up was negative on chest X-ray, neck CT and whole-body positron emission tomography/CT. Preoperative chemotherapy or radiation therapy was not administered due to the unwillingness of the elderly patient and consultation advice.

Intraoperatively, a new satellite lesion at 12 oclock and three in-transit metastases at 3 to 6 oclock were discovered. The satellite lesion was included within wide local excision of $2 \mathrm{~cm}$ safety margin and in-transit metastases were separately excised (Fig. 1). The zygomatic and buccal branches of facial nerve and parotid duct abutted the tumor and were sacrificed with partial en bloc excision of the parotid gland. Reconstruction was performed with a temporoparietal fascia transposition flap and full-thickness skin graft.

Microscopic examination revealed a well-differentiated SCC epithelial component and an undifferentiated sarcoma-like spindle cell mesenchymal component (Fig. 2). The mesenchymal component showed moderately pleomorphic features (spindle to round cytoplasms) with frequent mitoses. Some areas exhibited an obvious squamous to spindle cell transition, which excluded the possibility of collision of two separate tumors and suggested SpSCC or carcinosarcoma (Fig. 3). Immu-

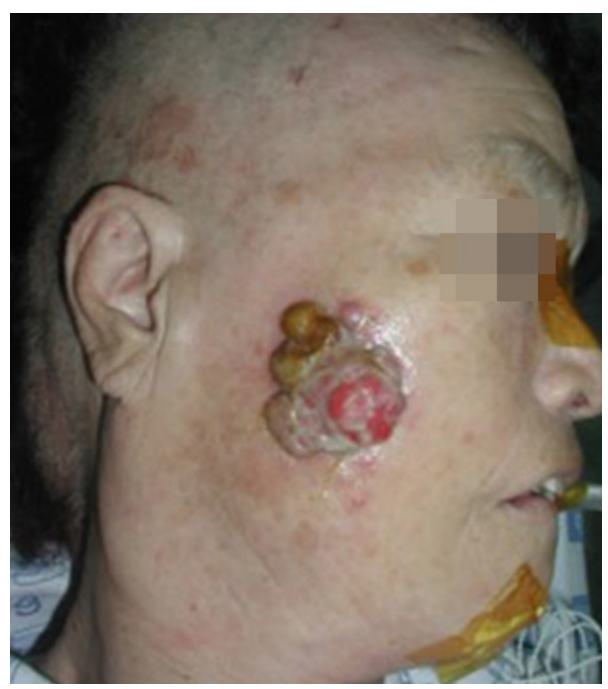

Fig. 1. A 77-year-old female patient presented with a nontender, rubbery, hard, protruding mass measuring $3 \times 3 \times 0.5 \mathrm{~cm}$ with ulcers and a satellite lesion at 12 oclock and three in-transit metastases at 3 to 6 oclock on the right cheek. nohistochemical staining in sarcomatous area were positive for pancytokeratin, $\mathrm{p} 40$, and vimentin, but negative for smooth muscle actin, desmin, S100, CD68, and HMB-45 (Fig. 4). These findings excluded carcinosarcoma and resulted in the diagnosis of SpSCC with mesenchymal metaplasia (Table 1). Lymphovascular invasion was present without perineural invasion. The free lateral and deep margins were estimated as $18 \mathrm{~mm}$ and 1 $\mathrm{mm}$ respectively. Microscopic findings of in-transit metastases showed metastatic tumors with a clear resection margin.

Starting on postoperative day 13 , dark red serous fluid was aspirated repeatedly from a preauricular fluid collection without

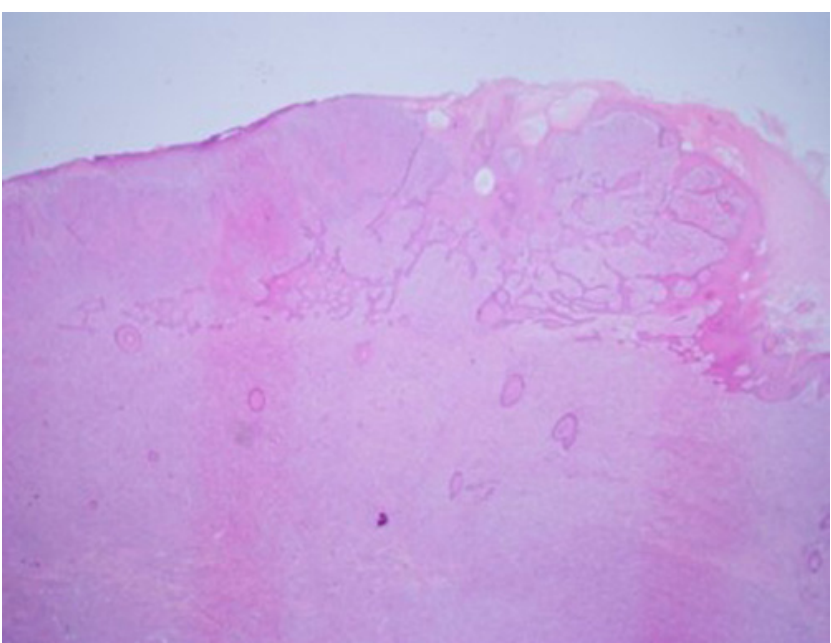

Fig. 2. Histopathological examination showing the biphasic malignant nature of tumor. The tumor had superficial epithelial and deep sarcomatous components. Squamous cell carcinoma of the epidermis infiltrated the upper dermis. The spindle tumor cells in the dermis and subcutis demonstrated atypical nuclei and frequent mitoses without any specific pattern of growth and cellular differentiation $(\mathrm{H} \& \mathrm{E}, \times 10)$.

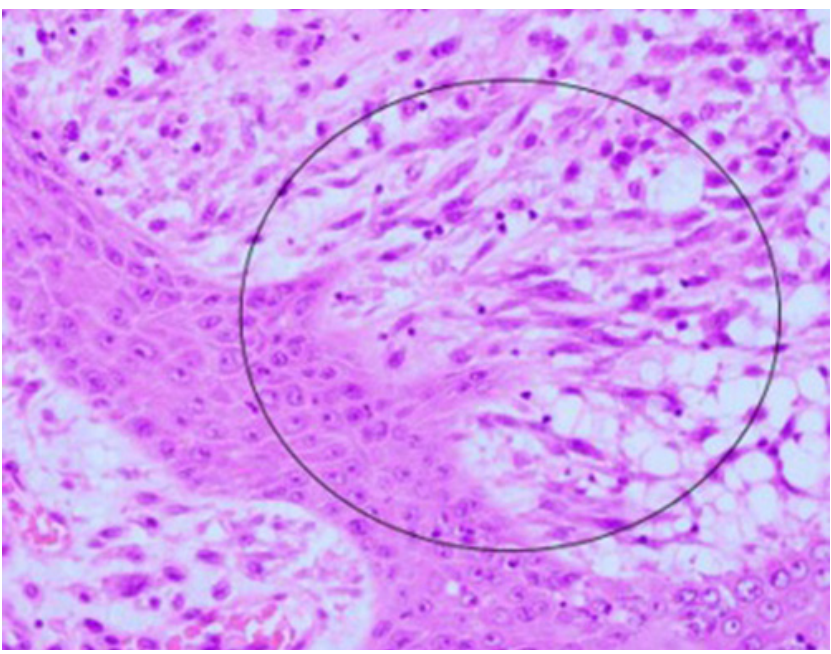

Fig. 3. A focal area showing squamous to spindle cell transition (H\&E, $\times 400)$. 

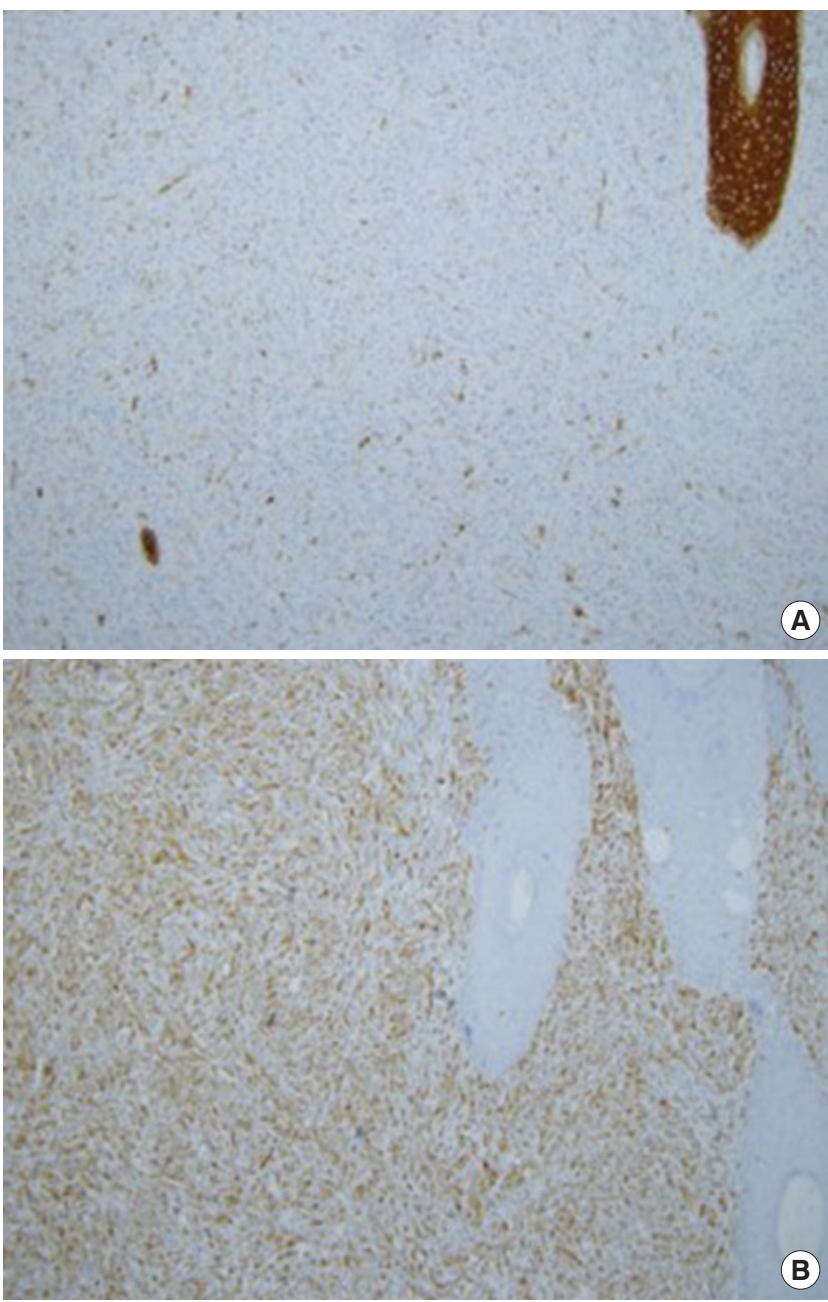

Fig. 4. Photomicrographs of immunohistochemical staining. (A) Pancytokeratin staining. The squamous cell component in the upper right corner strongly expressed pancytokeratin, and a few tumor cells in the spindle component also expressed pancytokeratin $(\times 100)$. (B) Vimentin staining. Tumor cells in the spindle cell component expressed vimentin strongly $(\times 100)$. malignant cells on cytology. Seventeen days after the initial operation, four in-transit metastases were excised at 5 to 6 oclock under local anesthesia (Fig. 5). Adjuvant radiation therapy was delayed until postoperative day 37 and then, after resolution of flap congestion and sialocele, radiation therapy was started to induce regression of a new $1-\mathrm{cm}$ mass at 8 oclock prior to a planned wide re-excision. Whether the recurrent mass was a remnant tumor or conduit spread along a nerve or a metastatic lymph node was unclear. The recurrent tumor shrunk initially but increased again during the 5-week radiation schedule (Fig. $6 \mathrm{~A})$. After $52 \mathrm{~Gy}$ of total radiation at the primary site, magnetic resonance imaging revealed a heterogenous mass measuring $3.8 \times 2 \mathrm{~cm}$ perforating the masseter deep to the mandible (Fig. $6 \mathrm{~B})$. The patient was transferred for re-excision and bone reconstruction. Multiple distant lung and adrenal metastases

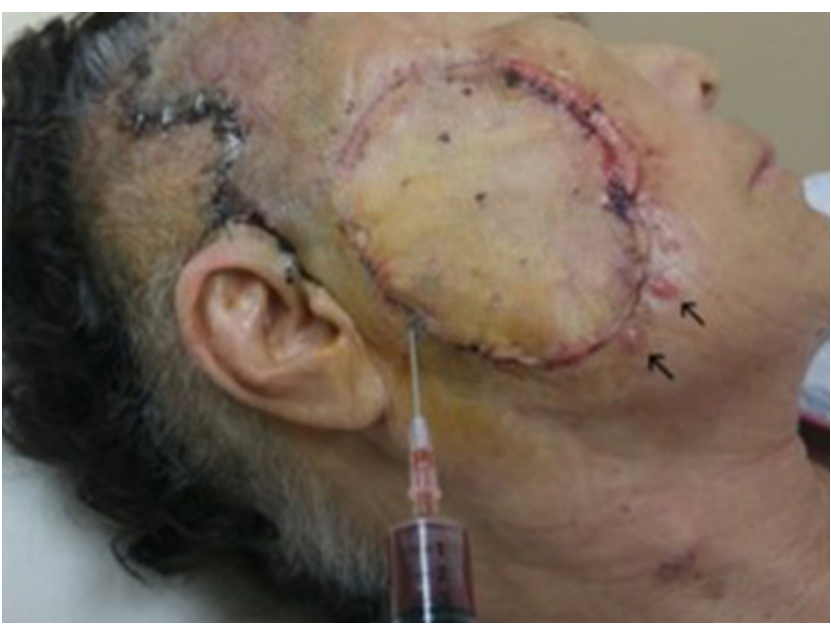

Fig. 5. Recurrent episodes of in-transit metastases. At postoperative day 13, during aspiration of preauricular sialocele, four more intransit metastases were clearly seen at 5 to 6 oclock (arrows).

Table 1. Immunohistochemical findings of common pleomorphic cutaneous spindle cell tumors ${ }^{\mathrm{a}}$

\begin{tabular}{|c|c|c|c|c|c|c|c|c|}
\hline Tumors/markers & $\begin{array}{c}\text { Cytokeratins } \\
\text { (wide-spectrum) }\end{array}$ & p40 & Vimentin & S100 protein & HMB-45 & SMA & Desmin & CD68 \\
\hline SpSCC & + & $+1-$ & + & - & - & - & - & - \\
\hline Spindle cell melanoma & $-1+$ & - & + & + & $-1+$ & - & $-1+$ & $+1-$ \\
\hline Atypical fibroxanthoma & - & - & + & - & - & $-1+$ & - & $+/-$ \\
\hline Leiomyosarcoma & $-1+$ & - & + & $-1+$ & - & + & $+/-$ & $-1+$ \\
\hline Angiosarcoma & $-1+$ & - & + & $-/+$ & - & - & - & $-1+$ \\
\hline This case (spindle cell component) ${ }^{\mathrm{b}}$ & Positive & Positive & Positive & Negative & Negative & Negative & Negative & Negative \\
\hline
\end{tabular}

+ , positive in more than $90 \%$ of cases; -, negative; +/-, positive in $50 \%$ to $75 \%$ of cases; and $-/+$, usually negative, but anomalous expression may be seen in up to $25 \%$ of cases.

a) Cytokeratins are epithelial markers and subclassified according to molecular weight (MW). Low-MW cytokeratins appear in simple one-layered epithelium in gland and duct. High-MW cytokeratins appear in complex epithelium, such as the uroepithelium and skin. Along with the epithelial to mesenchymal transition, spindle cell squamous cell carcinoma may lose or downregulate cytokeratins and acquire vimentin expression. Thus, a panel of antibodies including multiple cytokeratin antibodies based on microscopic findings is recommended; p40 is a highly specific epithelial marker, vimentin is a nonspecific mesenchymal cell marker, and S100 protein and HMB-45 are melanocytic markers. S100 is more sensitive and less specific. Smooth muscle actin (SMA) and desmin are markers of myogenic origin, and CD68 is a nonspecific marker of fibrohistiocytic differenti-

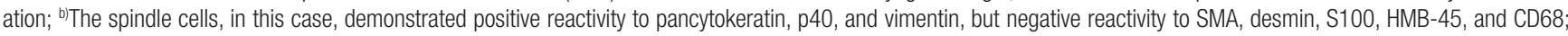
therefore, they featured epithelial markers without specific markers for any type of sarcoma, which excluded carcinosarcoma. 

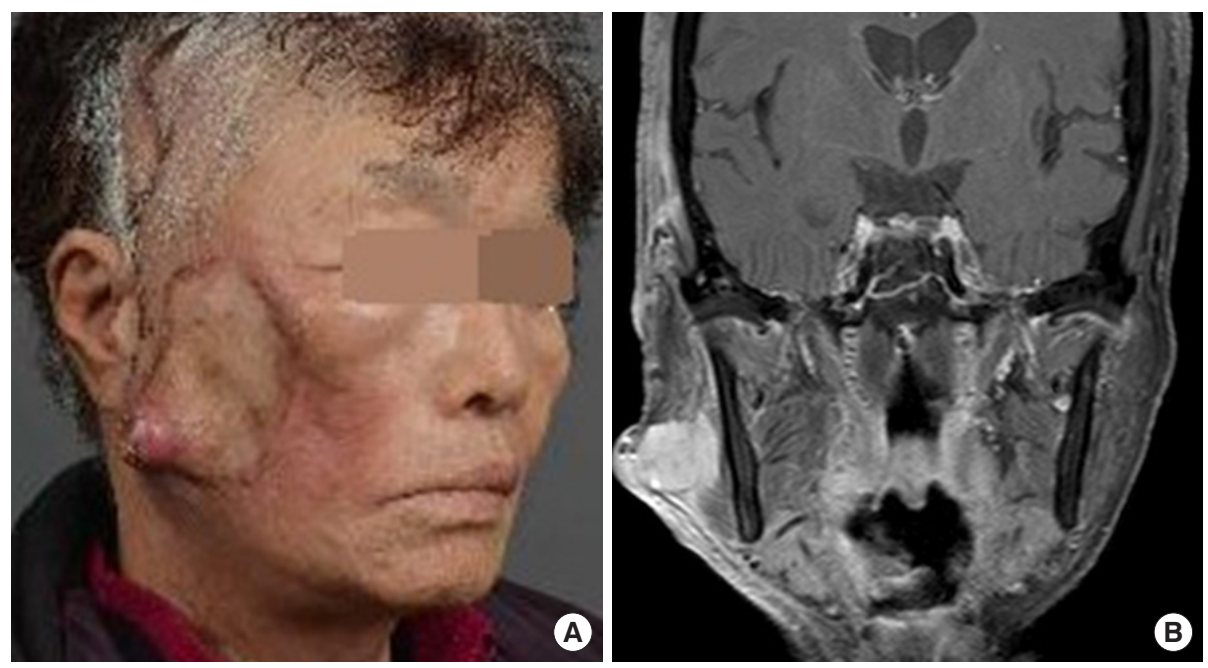

Fig. 6. Tumor recurrence. (A) Oblique photography on postoperative day 88. (B) Magnetic resonance imaging on postoperative day 76. A new mass measuring $3.8 \times 2 \mathrm{~cm}$ developed at the anterior aspect of the right parotid gland and perforated the masseter deep to the mandible ramus.

were discovered at the transferred hospital. The patient was lost to follow-up thereafter.

\section{DISCUSSION}

Cutaneous SCC (cSCC) has a high cure rate, 5-year disease-free rates of $91 \%$ or higher [12]. However, cSCC has many variants and management should be individualized. Among these variants, some chimeric biphasic combinations exist such as SpSCC, basosquamous cell carcinoma and carcinosarcoma, which are mixtures of SCC with sarcomatoid spindle cells, basal cell carcinoma, and true mesenchymal sarcoma, respectively.

Three common pathogenetic hypotheses have been proposed to explain these hybrid tumors [13-15]: (1) collision of two synchronous unrelated tumors (collision theory); (2) combination of two divergent cell lines from a common pluripotential stem cell (combination or divergence theory); and (3) conversion from a less aggressive carcinoma to a more aggressive carcinoma or sarcoma by metaplastic transformation (conversion theory).

Most studies now consider them as metaplastic epithelial carcinomas (conversion theory) because most such diseases have a monoclonal origin with the presence of a transition zone (Fig. 3) and are thought to represent late-onset squamatization of basal cell carcinoma in basosquamous cell carcinoma or the epithelial to mesenchymal transition (EMT) in SpSCC and carcinosarcoma [13-15].

EMT is a ubiquitous developmental process during organogenesis in which embryonic epithelial cells lose their features and acquire a mesenchymal phenotype (spindle, epithelioid, and pleomorphic cells). EMT is not just a developmental phenomenon in embryos, but is also a pathogenetic mechanism in metaplastic tumors and a well-known facilitator of metastasis and angiogenesis [16]. During EMT, differentiated epithelial cells lose their characteristics, including cell adhesion and polarity, reorganize their cytoskeleton, and acquire spindle cell morphology and the ability to migrate.

The main differential diagnoses for cutaneous spindle cell malignancies are spindle cell melanoma, atypical fibroxanthoma, pleomorphic dermal sarcoma, angiosarcoma, leiomyosarcoma and carcinosarcoma (Table 1) $[17,18]$. In this case, the obvious presence of SCC precluded those entities except carcinosarcoma. The initial misdiagnosis of carcinosarcoma was later corrected to SpSCC by further immunohistochemical analyses of spindle cells. These immunohistochemical findings also indirectly support EMT as the pathogenetic mechanism.

Therapeutically, recent articles have suggested that the prognosis of cutaneous SpSCC is the same as that of ordinary cSCCs [2] in contrast to previous views of SpSCC as a more aggressive variant [1] although without a high-level of evidence. However, other grave prognostic factors such as a size greater than $4 \mathrm{~cm}$, depth beyond the subcutaneous fat, abutment to nerve, and lymphovascular invasion placed this case as a high-risk lesion in National Comprehensive Cancer Network staging, T3 and stage III in the 8th edition of American Joint Committee on Cancer (AJCC-8) classification system, and T3 in the Brigham and Women's Hospital staging system [19,20].

In particular, in-transit metastases and satellitosis should raise a red flag for staging and management. They are frequently reported in melanoma, and their behavior in melanoma has been well investigated. According to the AJCC-8 cancer staging, both have as poor prognosis as nodal metastasis and belong to the $\mathrm{N}$ "c" subcategory and stage IIIB or higher [6,7]. As in-transit me- 
tastasis and satellitosis indicate advanced stages, the choice of local treatment does not affect survival, which is dictated by development of distant metastases. Microscopically, in-transit lesions appear as sharply circumscribed nodules of tumor cells with a clear demarcation between normal tissue. Therefore, the standard local treatment for in-transit metastasis is complete excision rather than wide excision for primary melanoma. A satellite lesion is best included in wide excision.

In non-melanoma cutaneous malignancies, in-transit metastasis and satellitosis are very rare and therefore, the prognostic consequences are unclear. They are not mentioned in AJCC-8 staging system for head and neck nonmelanoma skin cancer $[9,10,19]$. However, the 5-year survival rate of $13 \%$ in the largest series [8] and as these phenomena represent regional metastasis and aggressiveness of the primary disease, adjuvant radiation therapy for locoregional control and chemotherapy for prevention of regional and distant metastasis should be considered. In addition to conventional chemotherapy for cSCC using cisplatin and 5-fluorouracil, some promising new therapies such as epidermal growth factor receptor inhibitors including cetuximab and panitumumab and immune checkpoint inhibitor pembrolizumab-have been approved by the Food and Drug Administration or are under clinical trials for noncutaneous head and neck SCC [20].

A clear deep margin was achieved grossly, and on intraoperative frozen and postoperative permanent analyses. However, the excision might have been insufficient because of its narrowness $(1 \mathrm{~mm})$, the use of bread-loaf sectioning instead of Mohs micrographic surgery, and the possibility of discontinuous tumor extension. However, the inevitable sacrifice of the facial nerve forced us to lean toward postoperative radiation therapy, rather th an further surgical resection at the operating table. Unfortunately, flap congestion and sialocele delayed radiation therapy until tumor recurrence, which was followed by distant metastasis shortly.

\section{NOTES}

\section{Conflict of interest}

No potential conflict of interest relevant to this article was reported.

\section{Ethical approval}

The study was approved by the Institutional Review Board of Chungbuk National University Hospital (IRB No. 2009-11-003) and performed in accordance with the principles of the Declaration of Helsinki. Written informed consent was obtained.

\section{Patient consent}

The patient provided written informed consent for the publication and the use of her images.

\section{ORCID}

Eui-Tae Lee https://orcid.org/0000-0001-5525-8330

\section{REFERENCES}

1. Goldsmith LA, Katz SI, Gilchrest BA, Paller AS, Leffell DJ, Wolff K. Fitzpatrick's dermatology in general medicine. 8th ed. New York: McGraw-Hill; 2012.

2. Lee JH, Lee YB, Kim JW, Yu DS. Cutaneous spindle cell squamous cell carcinoma. Ann Dermatol 2015;27:113-5.

3. Kim YD, Suhr KB, Lee JH, Park JK. A clinical and histopathological study of cutaneous squamous cell carcinoma. Korean J Dermatol 2002;40:25-30.

4. Chi DH, Sung KJ, Koh JK. A clinical observation of primary epithelial skin cancers. Korean J Dermatol 1995;33:1085-90.

5. Hayes AJ, Clark MA, Harries M, Thomas JM. Management of in-transit metastases from cutaneous malignant melanoma. $\mathrm{Br}$ J Surg 2004;91:673-82.

6. Balch CM, Gershenwald JE, Soong SJ, Thompson JF, Atkins MB, Byrd DR, et al. Final version of 2009 AJCC melanoma staging and classification. J Clin Oncol 2009;27:6199-206.

7. Gershenwald JE, Scolyer RA, Hess KR, Sondak VK, Long GV, Ross MI, et al. Melanoma staging: evidence-based changes in the American joint committee on cancer eighth edition cancer staging manual. CA Cancer J Clin 2017;67:472-92.

8. Ma JH, Wu A, Veness M, Estall V, Hong A, Borg M, et al. Intransit metastasis from squamous cell carcinoma. Dermatol Surg 2016;42:1285-92.

9. Altunay I, Cerman AA, Sakiz D, Ates B. Marjolin's ulcer presenting with in-transit metastases: a case report and literature review. Ann Dermatol 2015;27:442-5.

10. Carucci JA, Martinez JC, Zeitouni NC, Christenson L, Coldiron B, Zweibel S, et al. In-transit metastasis from primary cutaneous squamous cell carcinoma in organ transplant recipients and nonimmunosuppressed patients: clinical characteristics, management, and outcome in a series of 21 patients. Dermatol Surg 2004;30:651-5.

11. Copcu E, Dikicioglu E, Sivrioglu N, Meteoglu I. Subcutaneous nodules on the face: acantholytic in-transit cutaneous metastasis. Dermatol Surg 2004;30:1415-9.

12. Rowe DE, Carroll RJ, Day CL Jr. Prognostic factors for local recurrence, metastasis, and survival rates in squamous cell carcinoma of the skin, ear, and lip: implications for treatment modality selection. J Am Acad Dermatol 1992;26:976-90. 
13. Lewis JE, Olsen KD, Sebo TJ. Spindle cell carcinoma of the larynx: review of 26 cases including DNA content and immunohistochemistry. Hum Pathol 1997;28:664-73.

14. Burston J, Clay RD. The problems of histological diagnosis in baso-squamous cell carcinoma of the skin. J Clin Pathol 1959; 12:73-9.

15. Bigby SM, Charlton A, Miller MV, Zwi LJ, Oliver GF. Biphasic sarcomatoid basal cell carcinoma (carcinosarcoma): four cases with immunohistochemistry and review of the literature. J Cutan Pathol 2005;32:141-7.

16. Ribatti D. Epithelial-mesenchymal transition in morphogenesis, cancer progression and angiogenesis. Exp Cell Res 2017; 353:1-5.

17. Compton LA, Murphy GF, Lian CG. Diagnostic immunohis- tochemistry in cutaneous neoplasia: an update. Dermatopathology (Basel) 2015;2:15-42.

18. Folpe AL, Cooper K. Best practices in diagnostic immunohistochemistry: pleomorphic cutaneous spindle cell tumors. Arch Pathol Lab Med 2007;131:1517-24.

19. Lydiatt WM, Patel SG, O’Sullivan B, Brandwein MS, Ridge JA, Migliacci JC, et al. Head and neck cancers-major changes in the American Joint Committee on cancer eighth edition cancer staging manual. CA Cancer J Clin 2017;67:122-37.

20. Work Group; Invited Reviewers, Kim JYS, Kozlow JH, Mittal B, Moyer J, et al. Guidelines of care for the management of cutaneous squamous cell carcinoma. J Am Acad Dermatol 2018;78: 560-78. 